\title{
Proteomic analysis of differently expressed proteins in sex differentiation phases of flower buds in monoecious Pistacia chinensis Bunge
}

\author{
Yifan Chen, Qian Bai, Funan Ruan and Shuchai Su* \\ Ministry of Education Key Laboratory of Silviculture and Conservation, College of Forestry, Beijing Forestry \\ University, 35 East Qinghua Road, Beijing, 100083, China
}

\begin{abstract}
The Pistacia chinensis Bunge is traditionally dioecious, and the female trees are more required to grow in practice for oil seed production. The discovery of monoecious $P$. chinensis Bunge in North China provided good raw materials to study the sex differentiation process. The objective of this study was to identify the differently expressed proteins in flower buds in two key sex differentiation phases in monoecious P. chinensis Bunge. Morphological observation and paraffin section were used to determine the key phenophases, and label-free quantitative technique was used for proteomic analysis. The results showed that the proteins related to oxidative stress resistance up-regulated while proteins involved in photosynthesis down-regulated during the female primordium differentiation in bisexual flower buds of the monoecious P. chinensis Bunge in early March, while proteins related to oxidative stress resistance, ribosome activity, and photosynthetic function up-regulated during the male primordium differentiation in bisexual flower buds of the monoecious $P$. chinensis Bunge in late May. The most up-regulated proteins all involved in the photosynthesis pathway in both kind of flower buds in late May compared to those in early March, and the down-regulated proteins all involved in the ribosome pathway. The identified differentially expressed proteins such as the $\mathrm{Cu} / \mathrm{Zn}$ superoxide dismutases may be possible molecular markers for sex determination in monoecious P. chinensis Bunge.
\end{abstract}

\section{ARTICLE HISTORY}

Received 17 July 2018

Accepted 8 April 2019

\section{KEYWORDS}

Flower bud;

label-free; monoecious

Pistacia chinensis

Bunge; proteomics;

sex differentiation

\section{Introduction}

Most flowering plants are monoecious, with flowers containing both male and female parts (Xiong et al. 2013). However, some economic forest tree species are dioecious, with only male or female flowers in individuals. The low yield of these species can be largely attributed to the unbalanced sex ratio of their flowers. Therefore, studies on sex determination mechanisms and sex ratio regulations in dioecious plants have always been of great concern, considering its important biological meanings and economic values.

It is believed that the sex determination of dioecious plants could be attributed to both genetic factors (e.g. sexual chromosomes, sexual determination genes) and environmental factors (e.g. light, temperature, $\mathrm{CO}_{2}$ ) (Milewicz and Sawicki 2012). Most dioecious plants such as maize (Zea mays), cucumber (Cucumis sativus) and watermelon (Citrullus lanatus) undergoes the development process of asexuality, bisexuality, and unisexuality. The sex determination takes place during the bisexual process, resulting from the development or degradation of the primordium of sex organs in flowers (Milewicz and Sawicki 2012). However, the key sexual determination genes have not been identified in any dioecious plants by now (Xiong et al. 2013).

The Chinese Pistacia tree (Pistacia chinensis Bunge) belongs to Pistacia genus (Anacardiaceae). It is widely planted in China and is the most preferred biodiesel tree (Wang 2011). The Pistacia tree is generally believed as strictly dioecious, therefore, the female plants are more required to grow in practice for oil seed production. Even though a few studies have been conducted on Pistacia grafting, hybridization, and molecular markers (Gercheva et al. 2008; Isfendiyaroglu and Ozeker 2009; Kafkas et al. 2003; Marra et al. 2007), the sex determination mechanisms of Pistacia is still unclear. In recent years, the monoecious $P$. chinensis has been discovered in North China (Wang 2015), providing

CONTACT Shuchai Su sushuchai@sohu.com

(c) Chen et al. 2019

This is an open access article distributed under the terms of the prevailing CC-BY license at the time of publication. 
good raw materials to study the sex differentiation and regulation of $P$. chinensis.

Proteomics is a powerful tool to analyze the protein profile quantitatively, which can provide fundamental understanding of the biological changes (Tsou et al. 2010). The label-free proteomic approach quantifies protein expression using multiple LC-MS/MS analyses directly without any labeling techniques. The protein quantification is based on either the peptide peak intensity or spectral count in chromatography, and changes in protein abundance are calculated via a direct comparison between different analyses (Zhu et al. 2010). Compared to the isotope or fluorescent labeling techniques, the label-free quantitative proteomic techniques can provide fast and low-cost measurements of protein expression in complex biological samples (Zhu et al. 2010). Therefore, it has been widely used in recent years in plant biology to explore the protein expression dynamics in conditions such as plant development or environmental stress (Szymanski et al. 2017; Wang et al. 2017; Zhang et al. 2017).

The relative stable sex phenotypes of female and bisexual flowers have been observed on monoecious $P$. chinensis by our research group in Tangxian (Hebei province, China) in the year of 2013, 2014 and 2015, while there was presence of fruits on some pure male inflorescences (Bai et al. 2016). This phenomenon took place within only 1 year, and has been confirmed using markers. Furthermore, the statistics showed that the overall trend in recent years was that the male branches turned into bisexual gender, with no female turning into male (Bai et al. 2016). Accordingly, these plants could be utilized to explore the underlying mechanisms of sex differentiation in P. chinensis.

The sex differentiation process usually starts from the asexual status, goes through the bisexual process and finally stays at this stage (monoecious plants) or enters into unisexual stage (dioecious plant) with the development or degradation of the primordium of sex organs. Based on the continuous observation by our research group starting from 2013, obvious and stable sex differentiation in monoecious $P$. chinensis plants takes place every year (Bai et al. 2016). The stamen primordium (male primordium) of the monoecious $P$. chinensis Bunge developed in late May after blooming, and differentiated to anthers to form male or bisexual flowers, while the stamen primordium in female flower buds degraded to form female flowers. Then the pistil primordium (female primordium) of the monoecious
P. chinensis Bunge in both bisexual flower buds and female flower buds developed in early March next year before blooming, and differentiated to the stigma and the ovary. Because the differentiation process of the stamen primordium in male flower buds in male tree and in bisexual flower buds in monoecious tree are similar (Bai et al. 2016), we may get some clues of the sex determination process in $P$. chinensis Bunge by comparing the bisexual flowers and female flowers in monoecious trees.

Therefore, the aim of this study was to identify and quantitate the differently expressed proteins between female $P$. chinensis and bisexual $P$. chinensis in sex differentiation phases of flower bud using label-free quantitative technique. Together with the undergoing genomic and transcriptomic studies, these data could provide some possible clues to depict the sex determination process in P. chinensis Bunge.

\section{Materials and methods}

\section{Plant materials}

The monoecious $P$. chinensis Bunge sampled in this study were located in Tangxian, Hebei province, China. The branches with stable sex phenotypes (female or bisexual flowers) in successive years were selected based on the previous record from our research group. Flower buds with different sex were identified through external morphological observation, and further confirmed through internal anatomical observation via optical microscope. Based on the continuous monitoring and observation, both female flower buds and bisexual flower buds (Fig. 1A, B) were sampled during two key phenophases for sex differentiation: (I) from May 27, 2016 to May 31, 2016 (labelled as 5F and 5B, respectively), and (II) from March 3, 2017 to March 7, 2017 (labelled as 3F and 3B, respectively). The male primordium in the bisexual flower buds developed during phenophase $\mathrm{I}$, while the female primordium in both the female flowers and the bisexual flowers developed during phenophase II. The male primordium that developed in the male flower buds went through the same differentiation process as that in bisexual flower buds (Bai et al. 2016), thus no male flower buds were sampled. Approximately $2 \mathrm{~g}$ of flower buds were randomly collected from 3 different trees for each sex phenotype in each phenophase, and the flower buds were kept in liquid nitrogen for further analysis. 


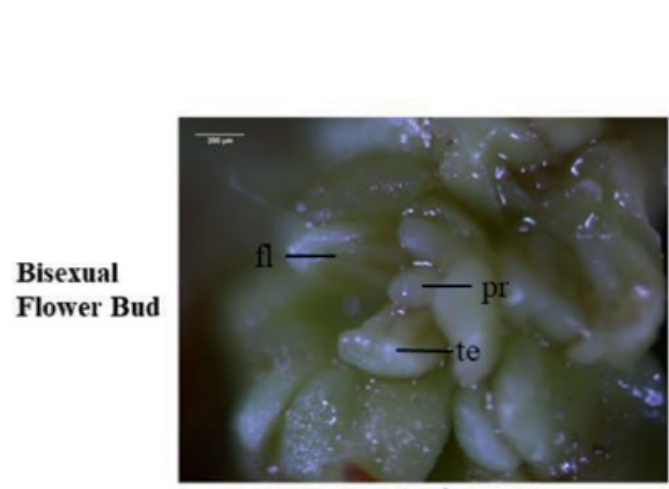

(A)

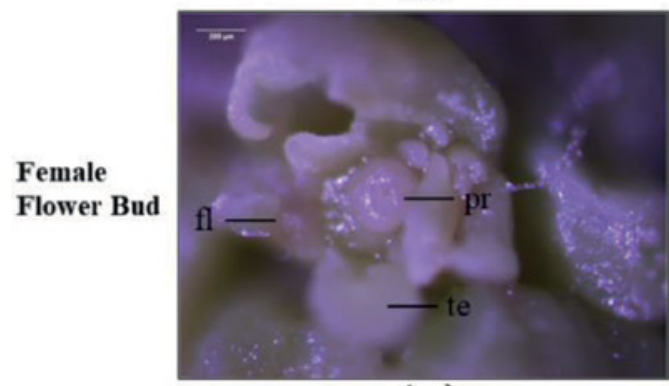

(B)

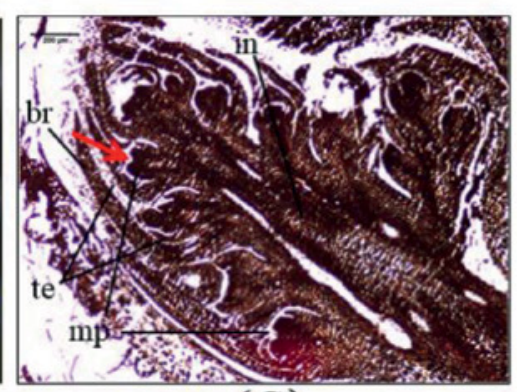

(C)

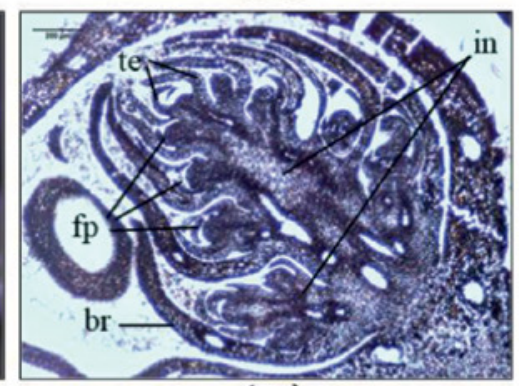

(D)
Early March, 2017

(phenophase II)

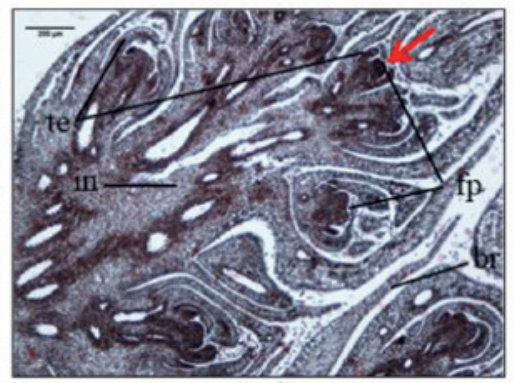

(E)

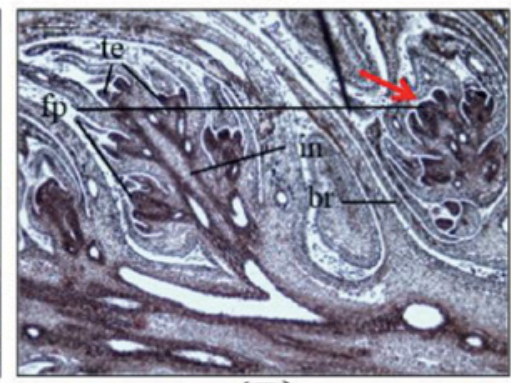

(F)

(G)

Phenophase I

Phenophase II

$\begin{array}{llcc} & \text { May 27, 2016 May 31, 2016 } & \text { March 3, 2017 } & \text { March 7, 2017 } \\ & & & \\ \text { Bisexual } & \text { male primordium developed } & \text { female primordium developed } \\ \text { Flower Bud } & \text { Sampled: 5B } & \text { Sampled: } 3 \mathrm{~B} \\ \text { Female } & \text { no primordium developed } & \text { female primordium developed } \\ \text { Flower Bud } & \text { Sampled: } 5 \mathrm{~F} & \text { Sampled: } 3 \mathrm{~F}\end{array}$

Figure 1. orphological changes of female and bisexual flower buds in different phenophases in paraffin sections. (A) Morphology of the bisexual flower bud. (B) Morphology of the female flower bud. (C) Paraffin sections of the bisexual flower bud in late May, 2016, when the male primordium in the bisexual flower bud started to develop. (D) Paraffin sections of the female flower bud before differentiation of the female primordiums in late May, 2016. (E) Paraffin sections of the bisexual flower bud in early March, 2017, when the female primordium in the bisexual flower bud started to develop. (F) Paraffin sections of the female flower bud in early March, 2017, when the female primordium started to develop. (G) Timelines of different phenophases and time-points for sample collection in this study. Abbreviations: br: bract; fl: floret; fp: female primordia; in: inflorecence axis; mp: male primordia; pr: primordia; te: tepal. Red arrows are representative male/female primordia developed in that phenophase. The scale represents $200 \mu \mathrm{m}$.

\section{Paraffin section of flower buds}

Several female and bisexual flower buds in different phenophases were selected and soaked in FAA solution $(6 \mathrm{~mL}$ formalin, $4 \mathrm{~mL}$ acetic acid, $90 \mathrm{~mL} 70 \%$ ethanol and $5 \mathrm{~mL}$ glycerin) for fixation about 48 hours. Then the fixed flower buds were dehydrated through a series of graded ethanol baths to displace the water, infiltrated with paraffin, and embedded into paraffin blocks. The flower buds in paraffin blocks were cut 
by microtome into $8-12 \mu \mathrm{m}$ sections, and then made into slides. The paraffin around the plant materials was washed out using a series of xylene and ethanol baths, and the flower buds were stained with $1 \%$ safranin and $\mathrm{PIHCH}$ successively. The flower buds sections were observed with OLYMPUS-BH microscope and pictures were captured using camera.

\section{Total protein extraction}

Approximately 0.5 to $1 \mathrm{~g}$ flower buds $(3 \mathrm{~F}, 3 \mathrm{~B}, 5 \mathrm{~F}$, or $5 \mathrm{~B})$ were separately ground and homogenized with a $-20^{\circ} \mathrm{C}$ pre-cooled mortar and pestle in the urea extraction buffer, which contained $150 \mathrm{mM}$ Tris- $\mathrm{HCl}$ (pH7.6), $8 \mathrm{M}$ urea, $0.5 \%$ SDS, 1.2\% Triton X-100, 20 mM EDTA, $20 \mathrm{mM}$ EGTA, $50 \mathrm{mM} \mathrm{NaF}, 1 \%$ glycerol 2-phosphate, $1 \mathrm{mM}$ PMSF, $5 \mathrm{mM}$ DTT, 0.5\% phosphatase inhibitor mixture 2 (Sigma), an EDTA-free protease-inhibitor mixture (Complete ${ }^{\mathrm{TM}}$ ), $5 \mathrm{mM}$ ascorbic acid, and $2 \%$ polyvinylpolypyrrolidone. The ratio of flower buds to urea extraction buffer was 1:3 (w/v). Plant cell debris were removed via centrifugation at $10,000 \mathrm{~g}$ for $1 \mathrm{~h}$ at $4{ }^{\circ} \mathrm{C}$ and then $110,000 \mathrm{~g}$ for $2 \mathrm{~h}$ at $13^{\circ} \mathrm{C}$. The protein supernatant was then mixed with 3 volumes of a cold acetone: methanol (12:1 v/v) organic mix for ureamethanol protein precipitation. The protein pellet was rinsed with 15 volumes $(\mathrm{v} / \mathrm{w}$ ) of a cold acetone: methanol: $\mathrm{H}_{2} \mathrm{O}$ mix (12:1:1.4 $\mathrm{v} / \mathrm{v}$ ) to remove residual pigment and urea precipitates. The protein pellet was air-dried and re-dissolved in a $1 / 3$ volume of resuspension buffer, which contains $50 \mathrm{mM}$ Tris- $\mathrm{HCl}(\mathrm{pH} 6.8), 8 \mathrm{M}$ urea, $5 \mathrm{mM}$ DTT, 1\% SDS, and $10 \mathrm{mM}$ EDTA. This was followed by another round of protein precipitation and re-suspension. The cycling of protein precipitation and resuspension was repeated twice. The resulting total protein content was measured via protein DC assay (Bio-Rad) and calculated according to a bovine serum albumin (BSA) protein standard curve.

\section{Protein digestion}

Digestion of protein were performed according to the procedures described by Zhu et al. (2013). Briefly, total protein were excised from the preparative tube and destained with $50 \mathrm{mM} \mathrm{NH}_{4} \mathrm{HCO}_{3}$ to the final concentration $0.5 \mathrm{mg} / \mathrm{mL}$. With the addition of $100 \mathrm{mmol} / \mathrm{L}$ DTT to its final concentration of $10 \mathrm{mmol} / \mathrm{L}$, the protein fractions were mixed at $56^{\circ} \mathrm{C}$ for $60 \mathrm{~min}$, then diluted 10x with $250 \mathrm{mmol} / \mathrm{L}$ lodoacetamide (IAM) and kept in dark for $60 \mathrm{~min}$. Finally, the samples were digested with trypsin (substrate to enzyme mass to mass ratio at $50: 1$ ) at $37^{\circ} \mathrm{C}$ for $12 \mathrm{~h}$. Digested supernatant fractions were stored at $-80^{\circ} \mathrm{C}$ without further treatment until MS analysis.

\section{Liquid chromatography (LC) - Tandem mass spectrometry (MS/MS) analysis}

Each sample loaded for LC-MS/MS was a mixture of 3 bio-replicates (plant materials from 3 different trees) of each kind of flower bud (3F, 3B, 5F, or 5B). Digested peptide mixtures from each sample were pressure-loaded onto a fused silica capillary column packed with 3- $\mu \mathrm{m}$ dionex C18 material (RP; Phenomenex). The RP sections with $100 \AA$ were $15 \mathrm{~cm}$ long, respectively, and the column was washed with buffer $A$ (water, $0.1 \%$ formic acid) and buffer $B$ (acetonitrile, $0.1 \%$ formic acid). After desalting, a 5-mm, 300- $\mu \mathrm{m}$ C18 capture tip was placed in line with an Agilent 1100 quaternary high performance liquid chromatography (HPLC) and analyzed using a 12-step separation. The first step consisted of a 5 -min gradient from $0 \%$ to $2 \%$ buffer $B$, followed by a 45 -min gradient to $40 \%$ buffer B. Next, the buffer B flowed by 3-min gradient from $40 \%$ to $80 \%$ and $10-$ min $80 \%$ buffer B. After 2-min buffer B gradient from $80 \%$ to $2 \%$, approximately $100 \mu \mathrm{g}$ of tryptic peptide mixture was loaded on to the columns and was separated at a flow rate of $0.5 \mu \mathrm{L} / \mathrm{min}$ by using a linear gradient. As peptides were eluted from the micro-capillary column, they were electrosprayed directly into a micro TOF-Q II mass spectrometer (BRUKER Scientific) with the application of a distal $180^{\circ} \mathrm{C}$ source temperature. The mass spectrometer was operated in the MS/MS (auto) mode. Survey MS scans were acquired in the TOF-Q II with the resolution set to a value of 20,000. Each survey scan $(50-2,500)$ was followed by five data-dependent tandem mass (MS/MS) scans at $2 \mathrm{HZ}$ normalized scan speed.

\section{Sequence database searching and data analysis}

Tandem mass spectra were analyzed using Proteome Discover $^{\mathrm{TM}}$ software (Thermo Scientific) against the NR_database_Sapindales_Genebank databases. The search results were then filtered using a cutoff of $1 \%$ for peptide false identification rate. Peptides with Z score $<4$ or Delta-Mass $>5$ ppm were rejected. Furthermore, the minimum number of peptides to identify a protein was set to 1 . The differentially expressed proteins (DEPs) were considered to be up-regulated if the fold 
change $(F C)$ was greater than 2 and down-regulated if the FC was less than 0.5 .

Bioinformatics analysis was carried out to categorize proteins based on biological processes, cellular component and molecular function using annotations in Protein Analysis Through Evolutionary Relationships (PANTHER) database v6.1 (www.pantherdb.org) (Thomas et al. 2003), which is in compliance with gene ontology (GO) standards. Signaling pathway analysis were performed with the tools on the Kyoto Encyclopedia of Genes and Genome (KEGG) database (http://www.genome.jp/kegg/pathway.html), respectively. Identified proteins in different groups were analyzed for molecular function, biological process or pathway term in PANTHER using the binomial test (Cho and Campbell 2000).

\section{Results}

\section{Morphological observation showed various differentiation processes of female and bisexual flower buds of monoecious P. chinensis trees}

In late April and early May, the flower buds on the monoecious $P$. chinensis trees started to grow and showed distinguished appearance (bigger but still green) from leaf buds. The morphological differentiation of flower buds began from the growing point which located at the middle bottom of the buds, and the bract, deputy panicle, secondary bract, secondary deputy panicle and floret primordium formed sequentially. During this period, there was no morphological differences between male primordium and female primordium. In late May (phenophase I), the sex differentiation emerged, with bulges arising around the floret primordium base. The bulges in the middle was treated as the female primordium (pistil primordium), while the bulges in the peripheral was treated as the male primordium (stamen primordium) (Bai et al. 2016). As the florets continued to develop, the pistil primordia sunk down (Fig. 1C, red arrow) and the stamen primordia began to differentiate in bisexual flower buds, whereas the stamen primordia in female buds degenerated into tepals (Fig. 1D). After phenophase I, internal structures of both flower buds were relative stable, thus entering into the dormancy stage. In early March next year (phenophase II), their dormancy was disrupted, with pistil primordia in both bisexual and female flower buds bulging (Fig. 1E, F, red arrows). As a result, the pistil primordia came into the differentiation stage. In early April, both flower buds entered into the blooming period. Therefore, female and bisexual flower buds of monoecious $P$. chinensis trees demonstrated various differentiation processes during phenophase I and phenophase II. More detailed description of the flower bud differentiation processes were included in another manuscript (Bai et al. submitted).

\section{Proteomic analysis of DEPs in both flower buds in different phenophases}

Through the label-free quantitative technique, a total of 3750 proteins was successfully identified in bisexual flower buds and female flower buds in two phenophases (Supplementary Table 1). Of these, 3267 $(87.1 \%)$ proteins were identified in the bisexual flower buds in two phenophases, while 3332 (88.9\%) proteins were identified in the female flower buds in two phenophases (Fig. 2A). When compared between $3 B$ and $3 F, 5 B$ and $5 F, 5 B$ and $3 B$, and $5 F$ and $3 F$, the number of DEPs classified are 516, 232, 806 and 637 , respectively, with 146, 128, 417, and 303 up-regulated proteins, and 370, 104, 389, and 334 down-regulated proteins, respectively (Fig. 2B). The $\mathrm{GO}$ analysis were performed to gain a better understanding of the DEPs involved in the biological process, cellular components, and molecular function in flower buds with different sex and in different phenophases. Compared $3 \mathrm{~B}$ to $3 \mathrm{~F}$, majority of DEPs were down-regulated and were annotated to cellular and metabolic process, molecular binding and cellular component (e.g. cell, cell part, membrane, organelle) (Fig. 3A). Compared 5B to 5F, majority of DEPs were annotated to cellular and metabolic process, cell and organelle components, and molecular binding and catalytic activity (Fig. 3B). Compared to $5 \mathrm{~B}$ and $3 \mathrm{~B}$, and $5 \mathrm{~F}$ and $3 \mathrm{~F}$, majority of DEPs were up-regulated and annotated to cellular and metabolic process, cellular component, and molecular binding and catalytic activity (Fig. 3C, D). In sum, the DEPs annotated to cellular and metabolic process, and molecular binding and catalytic activity accounted for approximately 60\% of all the DEPs. Moreover, a large proportion of these DEPs take functions in more than one process. Among these DEPs, 9 DEPs were involved in cellular or metabolic process only, 9 DEPs involved in molecular binding and catalytic activity only, while 43 DEPs involved in both processes (Table 1). Furthermore, copper/ zinc-superoxide dismutase was the most up-regulated DEPs when compared $3 \mathrm{~B}$ to $3 \mathrm{~F}$, and $5 \mathrm{~F}$ to $3 \mathrm{~F}$, whereas 
Table 1. Differentially expressed proteins involved in cellular/metabolic process or molecular binding/catalytic activity when compared bisexual and female flower buds in different phenophases.

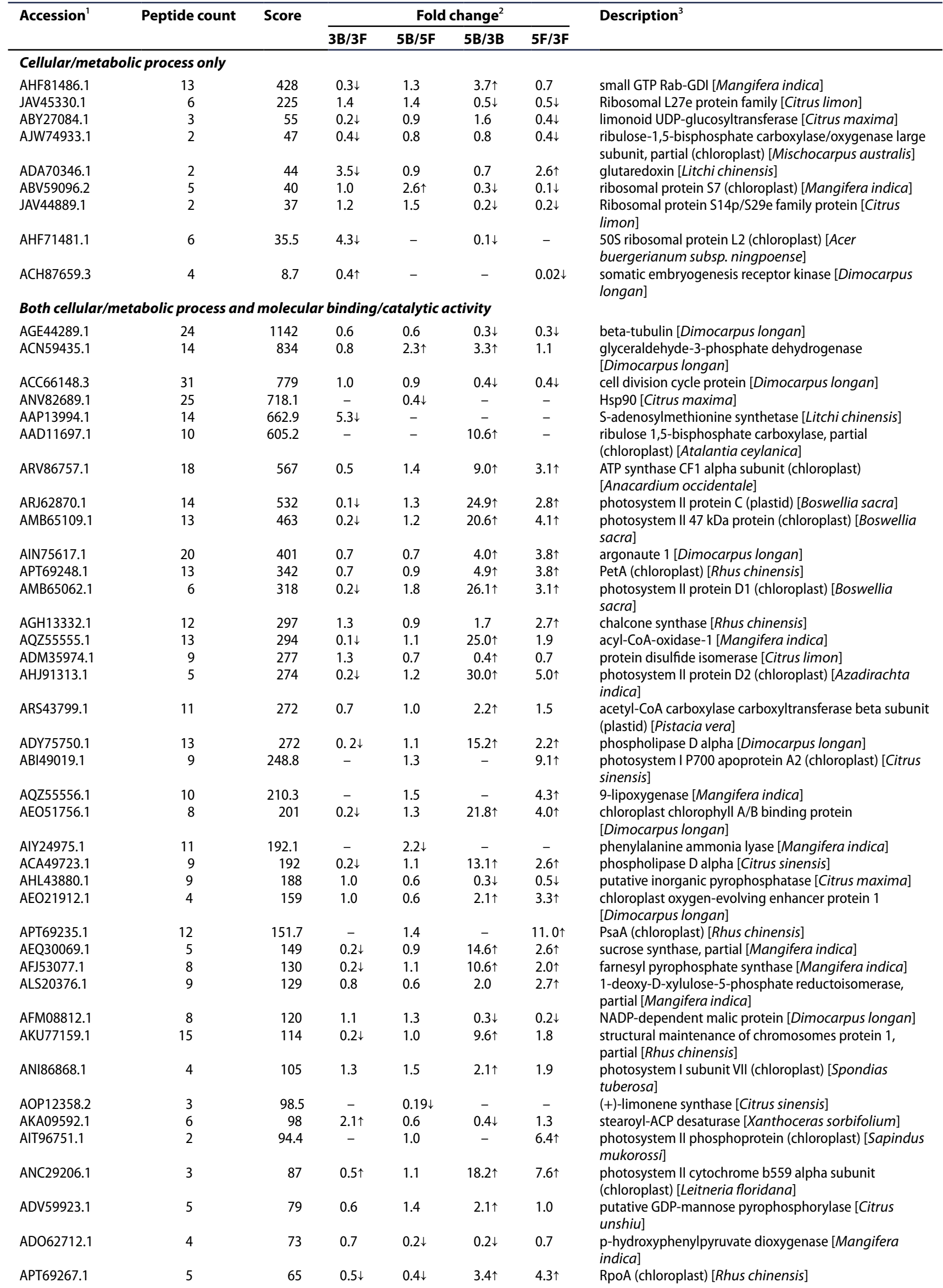




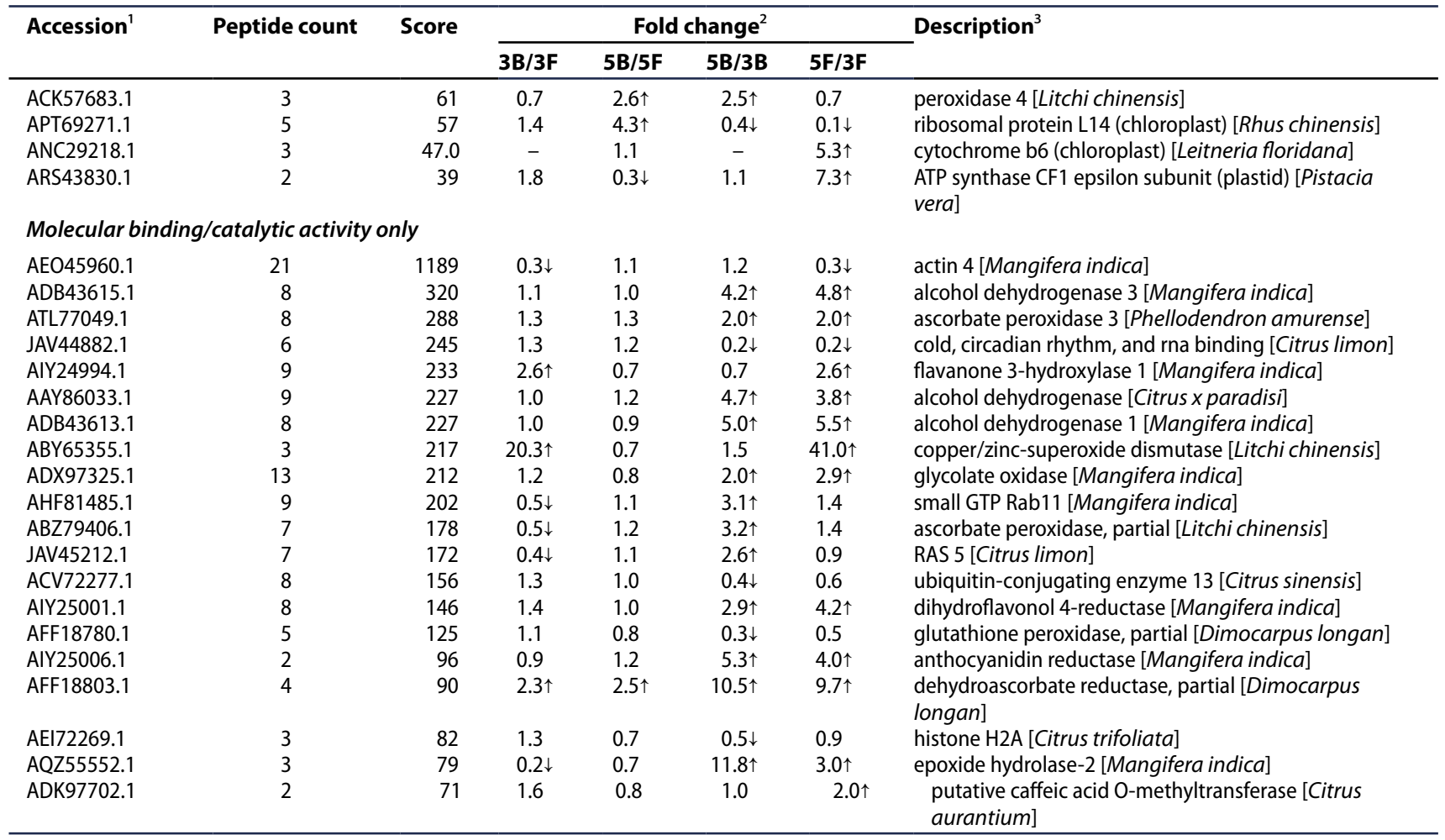

${ }^{1}$ Accession: accession number according to the NR_database_Sapindales_Genebank databases.

${ }^{2}$ 5B: bisexual flower buds sampled in late May, 2016; 3B: bisexual flower buds sampled in early March, 2017; 5F: female flower buds sampled in late May, 2016; 3F: female flower buds sampled in early March, 2017. $\uparrow$ : up-regulated proteins (fold-change $\geq 2.0$ ); $\downarrow$ : down-regulated proteins (foldchange $\leq 0.5$ ); -: not detected in either or both samples.

${ }^{3}$ Names in brackets ("[ ]") represent plant species.

ribosomal protein L14 (chloroplast) and photosystem II protein D2 (chloroplast) were the most up-regulated DEPs when compared $5 \mathrm{~B}$ to $5 \mathrm{~F}$, and $5 \mathrm{~B}$ to $3 \mathrm{~B}$, respectively. The most down-regulated DEPs comparing $3 B$ to $3 \mathrm{~F}, 5 \mathrm{~B}$ to $5 \mathrm{~F}, 5 \mathrm{~B}$ to $3 \mathrm{~B}$, and $5 \mathrm{~F}$ to $3 \mathrm{~F}$ were acyl-CoA-oxidase-1, (+)-limonene synthase, $50 S$ ribosomal protein L2 (chloroplast), and somatic embryogenesis receptor kinase, respectively (Table 1 ).

The KEGG pathway analysis revealed that photosynthesis and ribosome were two pathways that enriched in the most DEPs. In addition, other pathways that enriched in more than 10 DEPs included phenylpropanoid biosynthesis, flavonoid biosynthesis, alpha-linolenic acid metabolism, carbon metabolism, glutathione metabolism, glycolysis/gluconeogenesis, and phenylalanine metabolism (Fig. 4). A total of 13 DEPs participated in photosynthesis pathway, with all DEPs up-regulated when compared $5 B$ to $3 B$ and $5 \mathrm{~F}$ to $3 \mathrm{~F}$, and almost all DEPs down-regulated when compared $3 \mathrm{~B}$ to $3 \mathrm{~F}$ (Table 2). The photosystem II protein D2 (chloroplast) and PsaA (chloroplast) were the most up-regulated DEPs for $5 \mathrm{~B} / 3 \mathrm{~B}$ and $5 \mathrm{~F} / 3 \mathrm{~F}$ comparison, respectively, while photosystem II protein $C$ (plastid) was the most down-regulated DEPs for 3B/3F comparison in the photosynthesis pathway. A total of 9 DEPs participated in ribosome pathway, with all DEPs down-regulated when compared $5 \mathrm{~B}$ to $3 \mathrm{~B}$ and $5 \mathrm{~F}$ to $3 \mathrm{~F}$ (Table 2). The $50 \mathrm{~S}$ ribosomal protein L2 (chloroplast) and ribosomal protein L14 (chloroplast) were the most down-regulated DEPs when making this comparison in the ribosome pathway.

\section{Discussion}

Due to the lack of defined sex chromosomes, there is no reliable methods for sex determination of the dioecious $P$. chinensis plants prior to flowering. Moreover, the underlying sex determination mechanisms in $P$. chinensis are still unknown, making it difficult to breed female plants at juvenile stage for seed production (Xiong et al. 2013). The discovery of wild-type monoecious $P$. chinensis in China provided good materials to study the sex differentiation mechanisms of $P$. chinensis Bunge. The morphological and paraffin section observations in this study confirmed the sex differentiation process of monoecious $P$. chinensis trees reported by 
(A)

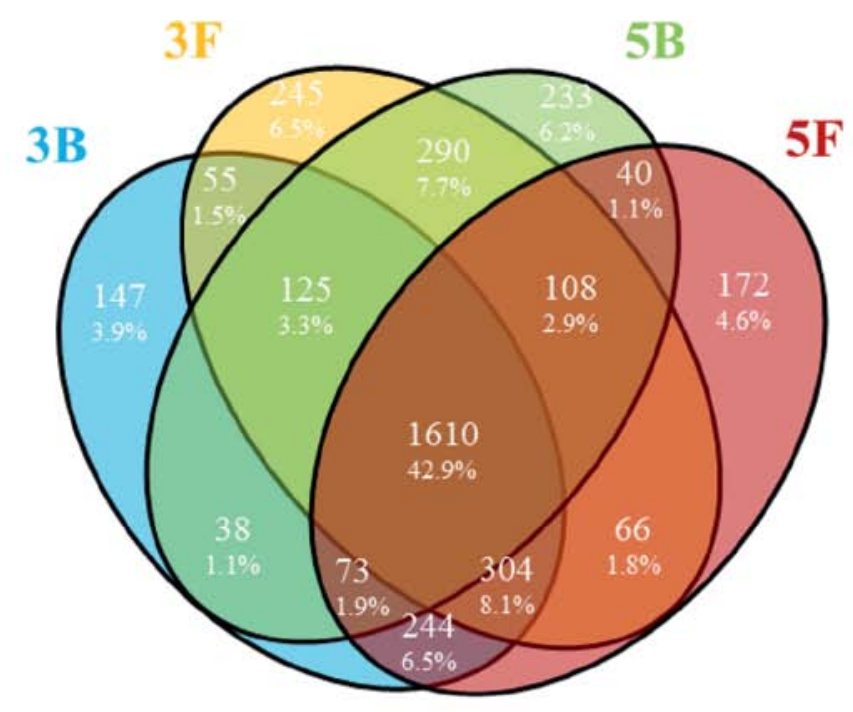

(B)

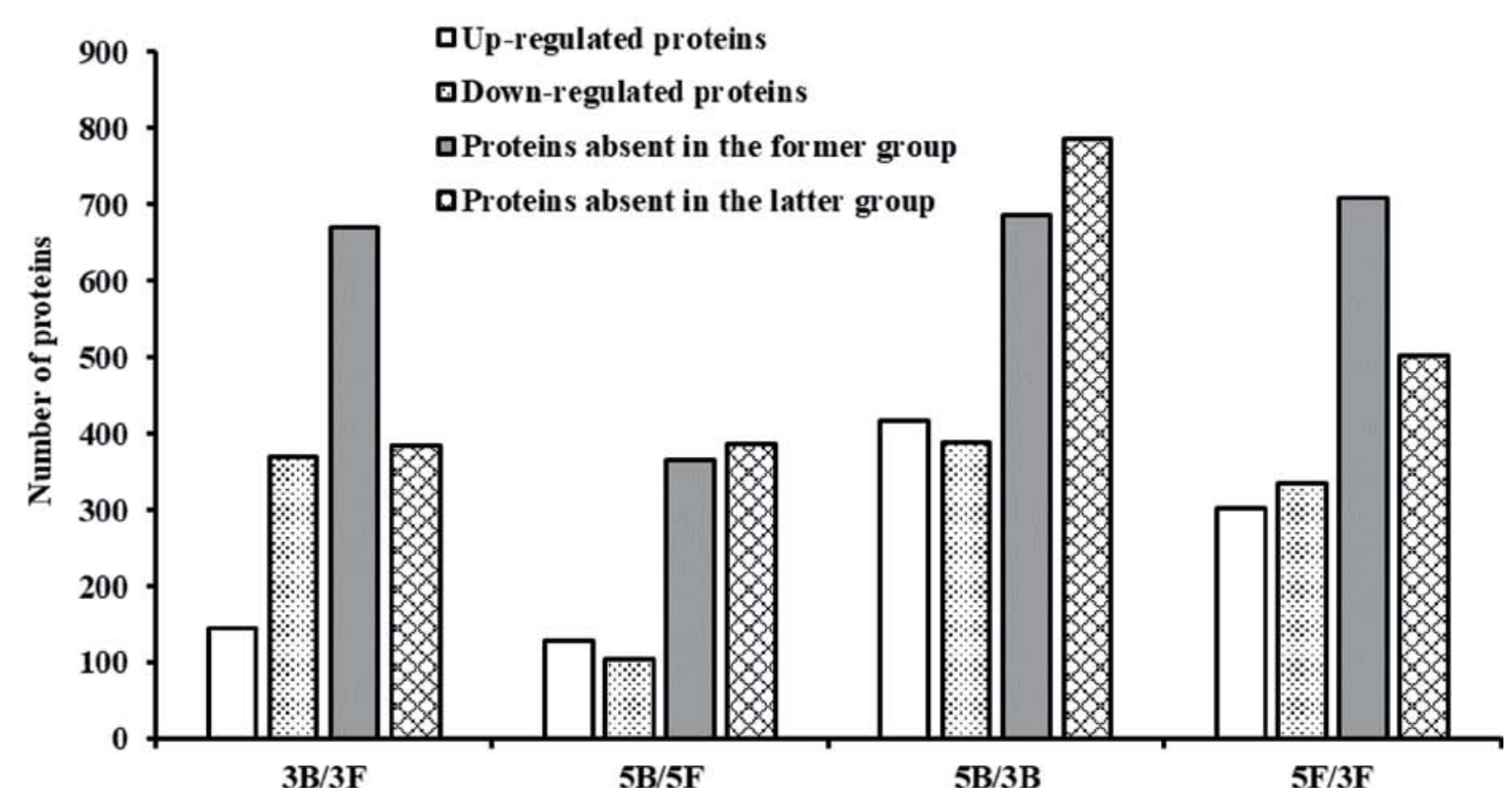

Figure 2. Distribution of identified proteins and differentially expressed proteins in bisexual flower buds or female flower buds in different phenophases. (A) Venn diagram showing the number of overlapping proteins that were identified in bisexual flower buds in late May, 2016 (5B) or in early March, 2017 (3B), and in female flower buds in late May, 2016 (5F) or in early March, 2017 (3F). (B) The number of differentially expressed proteins (up-regulated proteins with fold change $\geq 2.0$; down-regulated proteins with fold change $\leq 0.5$ ) and proteins not commonly expressed when compared among the four groups.

Bai et al. (2016). Therefore, the female and bisexual flower buds in late May 2016 (phenophase I) and early March 2017 (phenophase II) were sampled to study the difference during sex differentiation at the proteome level. Considering the male Pistacia chinensis Bunge tree is rare grown in the wild, and the sex phenotype of male flowers in sequential years were unstable, no male flower buds were collected in this study.

The label-free quantitative technique identified hundreds of DEPs when compared different flower buds in the same phenophase ( $3 \mathrm{~B}$ vs. $3 \mathrm{~F}$, and $5 \mathrm{~B}$ vs. $5 \mathrm{~F}$ ) or the same kind of flower bud in different phenophases $(5 B$ 
$3 B / 3 F$

(A)

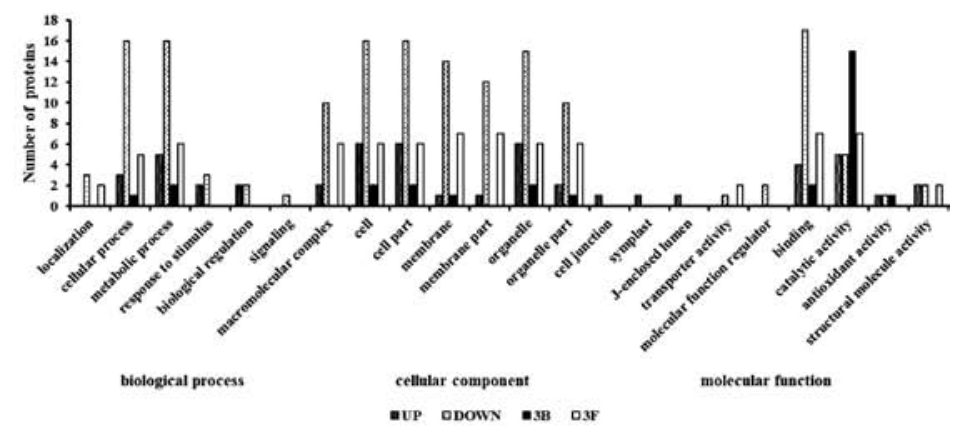

(B)

$5 B / 5 F$

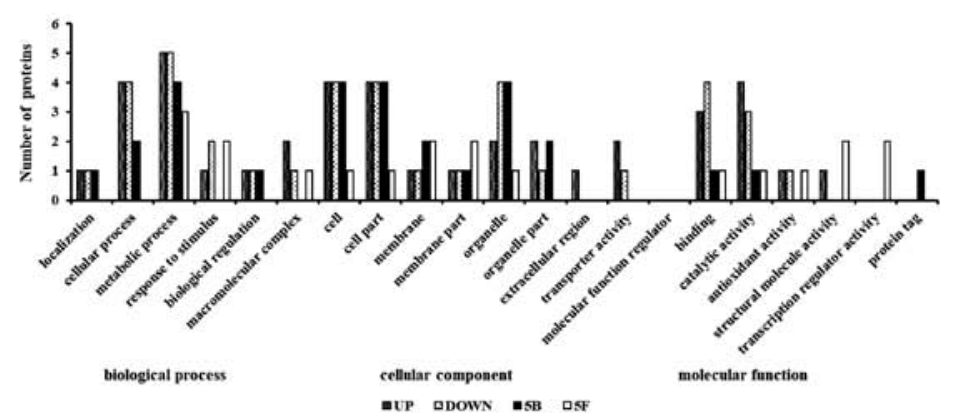

(C)

$5 B / 3 B$

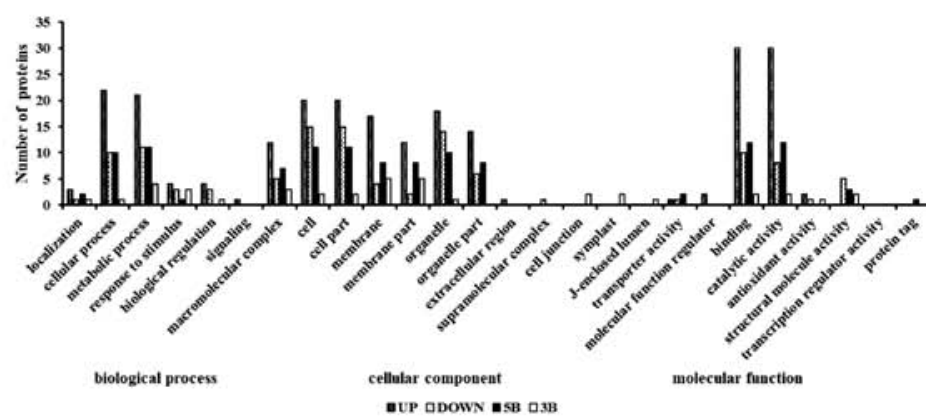

$5 F / 3 F$

(D)

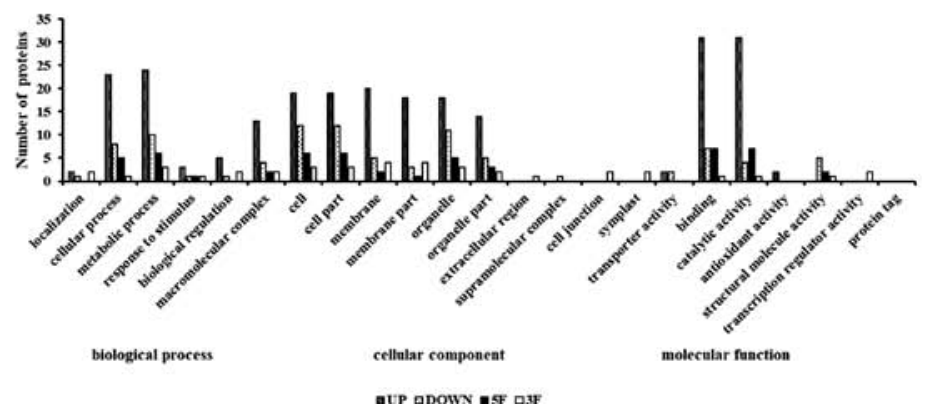

Figure 3. Gene Ontology (GO) analysis of differentially expressed proteins (DEPs) in bisexual flower buds or female flower buds in different phenophases. (A) GO analysis of DEPs between bisexual flower buds in early March, 2017 (3B) and female flower buds in early March, 2017 (3F). (B) GO analysis of DEPs between bisexual flower buds in late May, 2016 (5B) and female flower buds in late May, 2016 (5F). (C) $\mathrm{GO}$ analysis of DEPs between $5 \mathrm{~B}$ and $3 \mathrm{~B}$. (D) GO analysis of DEPs between $5 \mathrm{~F}$ and $3 \mathrm{~F}$. UP: up-regulated (fold change $\geq 2.0$ ); DOWN: downregulated (fold change $\leq 0.5$ ); $3 \mathrm{~B} / 3 \mathrm{~F} / 5 \mathrm{~B} / 5 \mathrm{~F}$ : expression only detected in $3 \mathrm{~B} / 3 \mathrm{~F} / 5 \mathrm{~B} / 5 \mathrm{~F}$. 


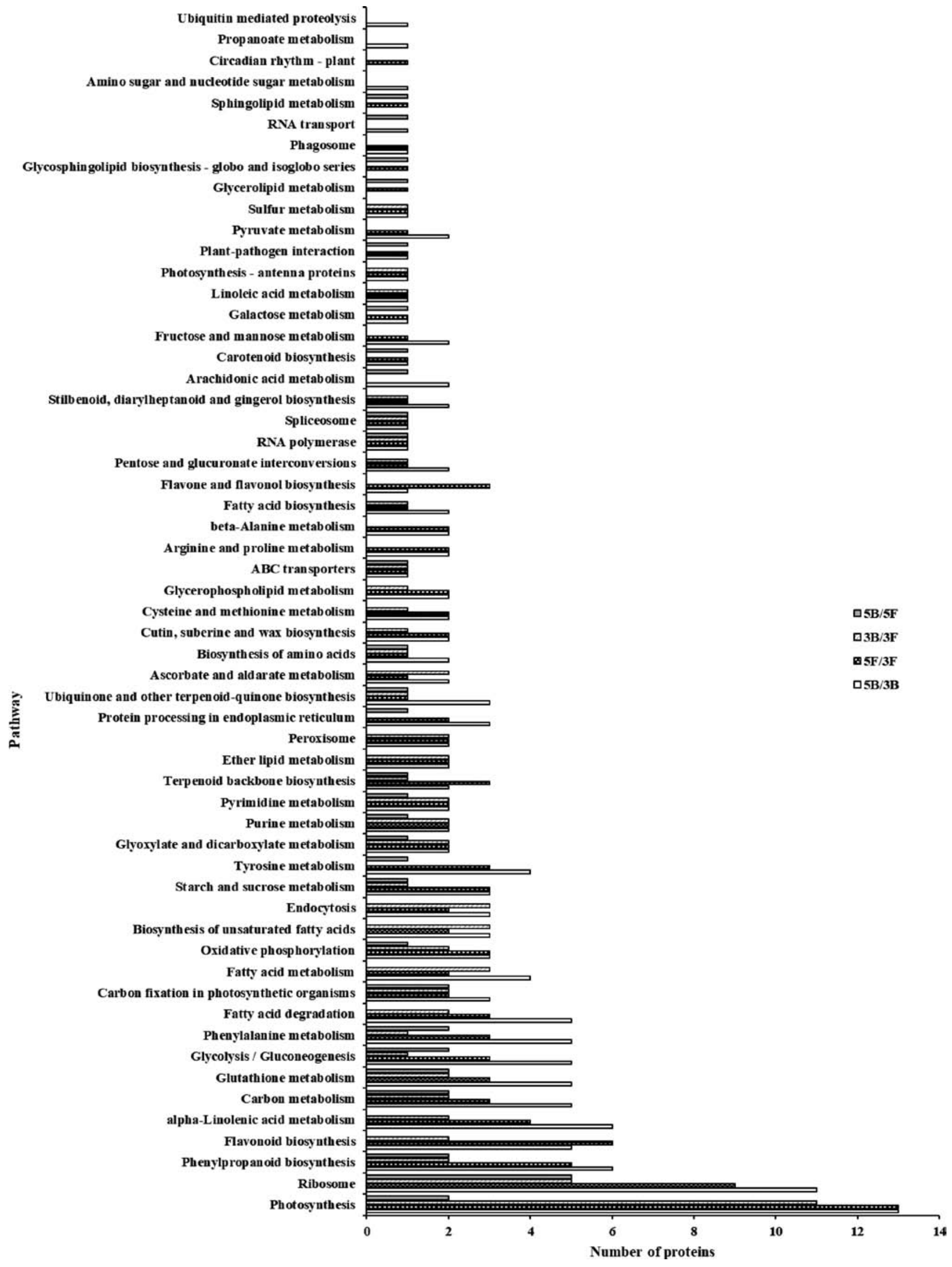

Figure 4. Kyoto Encyclopaedia of Genes and Genomes (KEGG) analysis of differentially expressed proteins in bisexual flower buds or female flower buds in different phenophases. 5B: bisexual flower buds sampled in late May, 2016; 3B: bisexual flower buds sampled in early March, 2017; 5F: female flower buds sampled in late May, 2016; 3F: female flower buds sampled in early March, 2017. 
Table 2. Differentially expressed proteins involved in major pathways when compared bisexual and female flower buds in different phenophases.

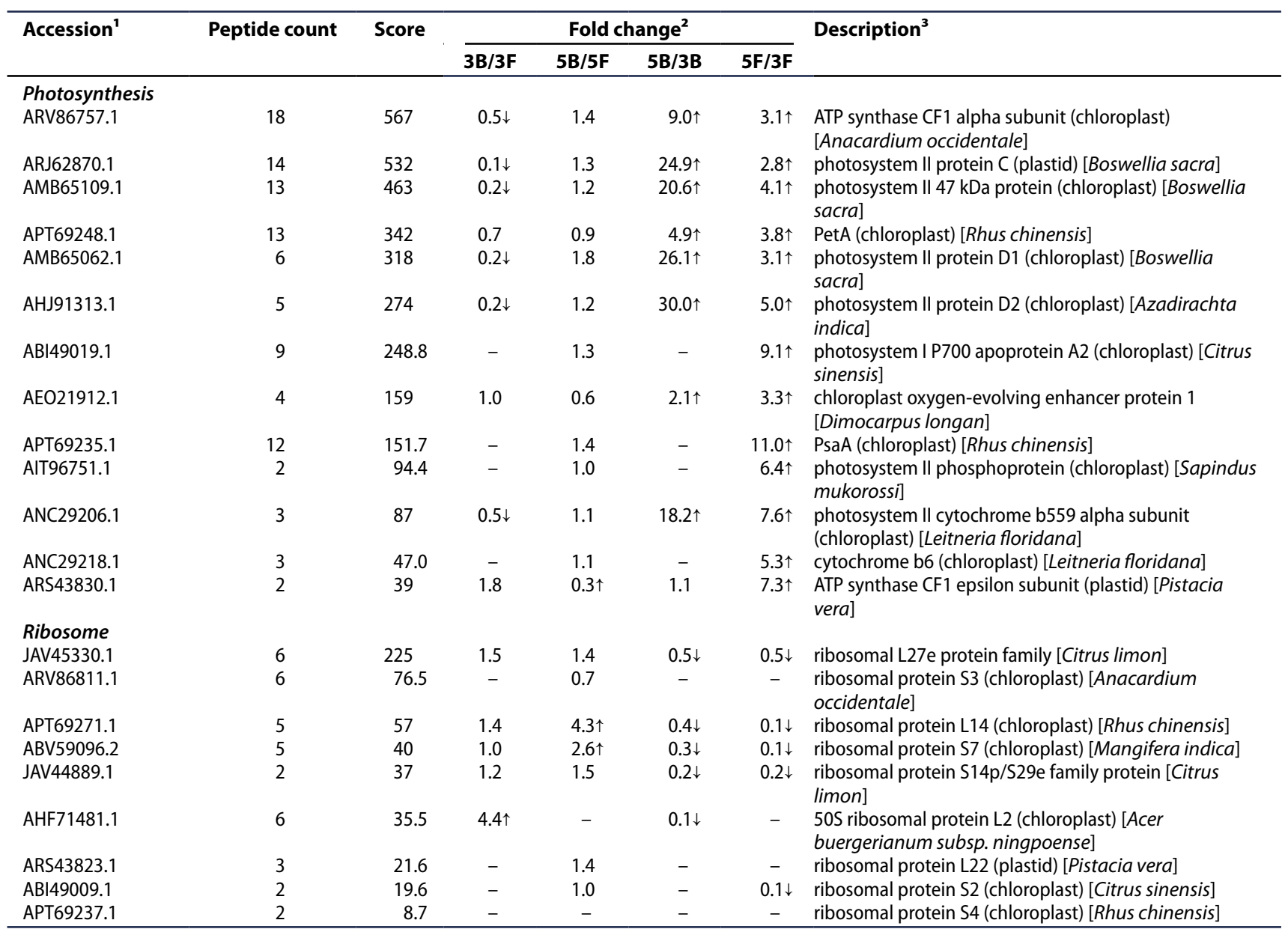

${ }^{1}$ Accession: accession number according to the NR_database_Sapindales_Genebank databases.

${ }^{2} 5 \mathrm{~B}$ : bisexual flower buds sampled in late May, 2016; 3B: bisexual flower buds sampled in early March, 2017; 5F: female flower buds sampled in late May, 2016; 3F: female flower buds sampled in early March, 2017. $\uparrow$ : up-regulated proteins (fold-change $\geq 2.0$ ); $\downarrow$ : down-regulated proteins (foldchange $\leq 0.5) ;-:$ not detected in either or both samples.

${ }^{3}$ Names in brackets ("[ ]") represent plant species.

the same kind of flower buds. The most up-regulated proteins all involved in the photosynthesis pathway in both kind of flower buds, and the down-regulated proteins all involved in the ribosome pathway, indicating the improved photosynthesis and reduced protein synthesis in late May compared to those in early March, which is also reasonable.

In this study, we reported the differently expressed proteins in key sex differentiation phases of flower buds in monoecious $P$. chinensis Bunge for the first time. The only previous literature that could be found on proteomic analysis of $P$. chinensis Bunge is from Xiong et al. (2013), who discovered that several functional and stress proteins change in abundance greatly between male dioecious $P$. chinensis and female dioecious $P$. chinensis, including NB-ARC domain containing protein
[Oryza sativa], light harvesting chlorophyll a/b-binding protein [Arabidopsis thaliana], temperature-induce lipocalin [Solanum lycopersicum], asorbate peroxidase (APX) [Pennisetum americanum], eukaryotic translation initiation factor 5A2 (elF-5A2) [Tamarix androssowii], and phosphoglycerate kinase 2 (PGK) [Vitis vinifera]. Similarly, we also observed the different expression of some stress-related proteins in this study. However, the two-dimensional electrophoresis approach was used for protein detection, and the green plants (NCBInr 20120922) database was used for DEPs searching in the study of Xiong et al. (2013), which may be not as accurate as ours from the methodology aspect.

The DEPs in flower buds with different sex and in various phenophases identified in this study could help to understand the mechanisms of sex differentiation 
vs. $3 \mathrm{~B}$, and $5 \mathrm{~F}$ vs. $3 \mathrm{~F}$ ) in P. chinensis. As the P. chinensis genome has not yet been sequenced, the identified proteins were searched against the database of Sapindales (P.chinensis Bunge belongs to Sapindales order) to identify the proteins of interest, as in other unsequenced plant species (Renuse et al. 2012). Large amount of proteins classified are from Mangifera indica (Mango), Citrus, Litchichinensis (Litchi), Dimocarpuslongan (Longan), Rhus chinensis (Sumac), etc. Majority of the identified DEPs involved in cellular/metabolic process or molecularbinding/catalytic activity, especially when compared between different phenophases, indicating that these biological and cellular processes changed drastically during the sex differentiation of P. chinensis Bunge.

During the key phase for female primordium differentiation (phenophase II), the most up-regulated proteins in bisexual flower buds included copper/ zinc-superoxide dismutase [Litchi chinensis] and Sadenosylmethionine synthetase [Litchi chinensis] compared to female flower buds. The plant $\mathrm{Cu} / \mathrm{Zn}$ superoxide dismutases (SODs) is one of the three SODs in plant which could catalyze the dismutation of superoxide radicals to oxygen and hydrogen peroxide, and plays a major role in the defense against toxic-reduced oxygen species, thus important for plant stress tolerance (Bowler et al. 1994). The S-adenosylmethionine (SAM) synthetase is an enzyme that creates SAM by reacting methionine and ATP (Horikawa et al. 1990). Meanwhile, the most down-regulated proteins in bisexual flower buds included acyl-CoA oxidase 1 [Mangifera indica] and epoxide hydrolases-2 [Mangifera indica] compared to female flower buds. Acyl-CoA oxidase was reported to be the key enzyme catalyzing the first step in the fatty acid $\beta$-oxidation pathway, and showed a positive relationship with the lactone formation in plant fruits (Zhang et al. 2017). Epoxide hydrolases could transform the epoxide-containing lipids to 1,2-diols by the addition of a molecule of water, and many of these oxygenated lipid substrates are associated with host defense responses (Morisseau 2013). Therefore, it seems that the female primordium differentiation in the bisexual flower bud of the monoecious $P$. chinensis Bunge in early March was associated with enhanced superoxide removal, and reduced fatty acid oxidation and epoxide-containing lipid removal in comparison to the female flower bud taken at the same time. Furthermore, our research also revealed that many proteins in the photosynthesis pathway were down-regulated during this process, e.g. photosystem II protein C (plastid), photosystem II protein D1 (chloroplast), photosystem II protein D2 (chloroplast), indicating the reduced photosynthesis function.

During the key phase for male primordium differentiation (phenophase I), the most up-regulated proteins in bisexual flower buds included ribosomal protein L14 (chloroplast) [Rhus chinensis], ribosomal protein S7 (chloroplast) [Mangifera indica], peroxidase 4 [Litchi chinensis], glyceraldehyde-3-phosphate dehydrogenase (GAPDH) [Dimocarpus longan] and dehydroascorbate reductase (DHAR) [Dimocarpus longan] compared to female flower buds, while the most down-regulated proteins was (+)-limonene synthase [Citrus sinensis]. Peroxidase was reported to be responsible for eliminating peroxides to increase the plant's defenses against pathogens (Karthikeyan et al. 2005). The GAPDH was usually involved in the glycolysis pathway, and the inactivation of GAPDH would lead to the consequences of oxidative stress (Hwang et al. 2009). The DHAR could regulate the cellular ascorbic acid redox state, which in turn affects cell responsiveness and tolerance to environmental stress, and increased DHAR expression maintained higher levels of photosynthetic functioning, resulting in delayed leaf aging (Chen and Gallie 2006). The (+)-limonene synthase is one of the synthases for limonene, a kind of terpenes that serve as chemical defense agents against microbial infection and predation and as attractants for pollinators (Pichersky and Gershenzon 2002). To summarize, the male primordium differentiation in the bisexual flower bud of the monoecious $P$. chinensis Bunge in late May was associated with higher oxidative stress resistance, the ribosome activity, and the possible enhanced photosynthetic function in comparison to the female flower bud taken at the same time.

When compared the above two processes, the increased expression of oxidative stress-resistance proteins during both female and male primordium differentiation were observed. This can support the theory of Horandl and Speijer (2018), who stated that in the evolutionary order of events, repair of oxidative damage was the first step as a response to endogenous reactive oxygen species production in eukaryotes, and this happens during prophase I of meiosis, the most indispensable phase of sex. On the other hand, the opposite expression pattern of photosynthesisrelated proteins during these two processes could be attributed to the differences in light and temperature between the differentiation phases.

To study the effect of sex differentiation phases, the DEPs in different phenophases were also compared on 
of flower buds in monoecious Pistacia chinensis Bunge. Moreover, the DEPs could act as molecular markers for sex determination. Many sex-linked molecular markers such as AFLP, RAPD, RFLP and microsatellite have been generated for sex determination in dioecious plants. However, these techniques need to design and test different sets of random primers and the function of amplified products is often unclear (Esfandiyari et al. 2012), while the proteins classified through proteomic analysis could be quantified directly with the function known. Therefore, our research results may have certain prospects in application.

\section{Conclusion}

Our study identified the differently expressed proteins in flower buds in two key sex differentiation phenophases in monoecious $P$. chinensis Bunge. In the bisexual flower bud of the monoecious $P$. chinensis Bunge, the female primordium differentiation in early March was associated with enhanced oxidative stress resistance and reduced photosynthesis function, while the male primordium differentiation of $P$. chinensis Bunge in late May was associated with increased oxidative stress resistance, the ribosome activity, and the enhanced photosynthetic function, in comparison with the female flower bud taken at the same time. The identified differentially expressed proteins such as the $\mathrm{Cu} / \mathrm{Zn}$ superoxide dismutases could act as a possible molecular marker for sex determination in monoecious $P$. chinensis Bunge. With the results of the undergoing genomic and transcriptomic studies, this proteomic study may help to depict the underlying sex determination mechanisms of $P$. chinensis Bunge.

\section{Acknowledgements}

This work was supported by the Fundamental Research Funds for the Central Universities (2015ZCQLX-02). In addition, we thank Dr. Shuai Zhang (China Agricultural University, Beijing, China) for critically revising this manuscript, and thank Beijing BangFei Bioscience Company for their help on proteomic analysis.

\section{Supplementary material}

Supplementary material is available online at: https://doi.org/10.6084/m9.figshare.8229137

\section{References}

Bai, Q., Su, S., Lin, Z., Leng, P., Wang, W. (2016). The variation characteristics and blooming phenophase of monoecious Pistacia chinensis Bunge. Hort. Sci. 51:961-967.

Bai et al. (submitted). Sex determination during inflorescence bud differentiation in monoecious Pistacia chinensis Bunge. Trees.

Bowler, C., Camp, W.V., Montagu, M.V., Inze, D. (1994). Superoxide dismutase in plants. Crit. Rev. Plant Sci. 13:199-218.

Chen, Z., Gallie, D.R. (2006). Dehydroascorbate reductase affects leaf growth, development, and function. Plant Physiol. 142:775-787.

Cho, R.J., Campbell, M.J. (2000). Transcription, genomes, function. Trends Genet. 16: 409-415.

Esfandiyari, B., Davarynejad, G.H., Shahriari, F., Kiani, M., Mathe, A. (2012). Data to the sex determination in Pistacia species using molecular markers. Euphytica. 185:227-231.

Gercheva, P., Zhivondov, A., Nacheva, L., Avanzato, D. (2008). Transsexual forms of pistachio (Pistacia terebinthus L.) from Bulgaria-biotechnological approaches for preservation, multiplication and inclusion in selection programs. Bulg. J. Agr. Sci. 14:449-453.

Horandl, E., Speijer, D. (2018). How oxygen gave rise to eukaryotic sex. Proc. R. Soc. B. 285: 20172706.

Horikawa, S., Sasuga, J., Shimizu, K., Ozasa, H., Tsukada, K. (1990). Molecular cloning and nucleotide sequence of CDNA encoding the rat kidney S-adenosylmethionine synthetase. J. Biol. Chem. 265:13683-13686.

Hwang, N.R., Yim, S.H., Kim, Y.M., Jeong, J., Song, E.J., Lee, Y.J., Lee, J.H., Choi, S., Lee, K.J. (2009). Oxidative modifications of glyceraldehyde-3-phosphate dehydrogenase play a key role in its multiple cellular functions. Biochem. J. 423:253-264.

Isfendiyaroglu, M., Ozeker, E. (2009). Inflorescence features of a new exceptional monoecious Pistacia atlantica Desf. (Anacardiaceae) population in the barbaros plain of Izmir/Turkey. Intl. J. Plant. Prod. 3:93-98.

Kafkas, S., Acar, I., Gozel, H. (2003). A project on developing monoecious pistachio (Pistacia vera $L$.) populations and determination of sex mechanism in pistachio. XIII GIEMPA Meeting on Almonds and Pistachios. Options Mediterraneennes. 63:57-60.

Karthikeyan, M., Jayakumar, V., Radhika, K., Bhaskaran, R., Velazhahan, R., Alice, D. (2005). Induction of resistance in host against the infection of leaf blight pathogen (Alternaria palandui) in onion (Allium cepa var aggregatum). Indian J. Biochem. Bio. 42:371-377.

Marra, F.P., Vaccaro, A., Avanzato, D., Meli, M., Zhivondov, A., Buffa, R., Caruso, T. (2007). Phenological and morphological studies of Pistacia terebinthus $L$. genotypes native of Bulgaria with different asset of tree sexuality. Balkan Symposium on Fruit Growing. 825:63-70.

Milewicz, M., Sawicki, J. (2012). Mechanisms of sex determination in plants. Cas. Slez. Muz. Opava. (A). 61:123-129.

Morisseau, C. (2013). Role of epoxide hydrolases in lipid metabolism. Biochimie. 95:91-95.

Passamani, L.Z., Barbosa, R.R., Reis, R.S., Heringer, A.S., Rangel, P.L., Santa-Catarina, C., Grativol, C., Veiga, C.F.M., Souza-Filho, 
G.A., Silveira, V. (2017). Salt stress induces changes in the proteomic profile of micropropagated sugarcane shoots. PLoS ONE. 12:e0176076.

Peleman, J., Boerjan, W., Engler, G., Seurinck, J., Botterman, J., Alliotte, T., Montagu, M.V., Inze, D. (1989). Strong cellular preference in the expression of a housekeeping gene of arabidopsis thafiana encoding S-adenosylmethionine synthetase. The Plant Cell. 1:81-93.

Pichersky, E., Gershenzon, J. (2002). The formation and function of plant volatiles: perfumes for pollinator attraction and defense. Plant Bio. 5:237-243.

Renuse, S., Harsha, H.C., Kumar, P., Acharya, P.K., Sharma, J., Goel, R., Sameer Kumar. G.S., Raju, R., Keshava Prasad, T.S., Slotta, T., Pandey, A. (2012). Proteomic analysis of an unsequenced plant-Mangifera indica. J Proteomics. 75:5793-5796.

Szymanski, J., Levin, Y., Savidor, A., Breitel, D., Chappell-Maor, L., Heinig, U., Topfer, N., Aharoni, A. (2017) Label-free deep shotgun proteomics reveals protein dynamics during tomato fruit tissues development. The Plant J. 90:396-417.

Thomas, P.D., Campbell, M.J., Kejariwal, A., Mi, H., Karlak, B., Daverman, R., Diemer, K., Muruganujan, A., Narechania, A. (2003). PANTHER: a library of protein families and subfamilies indexed by function. Genome Res. 13: 2129-2141.

Tsou, C.C., Tsai, C.F., Tsui, Y.H., Sudhir, P.R., Wang, Y., Chen, Y., Chen, J.Y., Sung, T.Y., Hsu, W.L. (2010). IDEAL-Q: an automated tool for label-free quantitation analysis using an efficient peptide alignment approach and spectral data validation. Mol. Cell Proteomics. 9:131-144.
Wang, C., Wang, J., Wang, X., Xia, Y., Chen, C., Shen, Z., Chen, Y. (2017). Proteomic analysis on roots of Oenothera glazioviana under copper-stress conditions. Sci. Rep. 7:10589.

Wang, X., Zhang, C., Yuan, M. (2011) The current situation of the development of Pistacia chinensis in HeBei Province. J. HeBei For. Sci. Technol. 6(3):56-58.

Wang, W., He, H., Bai, Q., Qi, P., Su, S., Fu, X., Chen, F. (2015). Hermaphroditism and fertility in Pistacia chinensis bunge. ISHS Acta. Hort. 1074:129-133.

Xiong, E., Wu, X., Shi, J., Wang, X., Wang, W. (2013). Proteomic identification of differentially expressed proteins between male and female plants in Pistacia chinensis. PLOS ONE. 8: e64276.

Zhang, L., Li, Ha., Gao, L., Qi, Y., Fu, W., Li, X., Zhou, X., Gao, Q., Gao, Z., Jia, H. (2017). Acyl-CoA oxidase 1 is involved in $\gamma$-decalactone release from peach (Prunus persica) fruit. Plant Cell Rep. 36:829-842.

Zhang, N., Zhang, H., Sun, Q., Cao, Y., Li, X., Zhao, B., Wu, P., Guo, $Y$. (2017). Proteomic analysis reveals a role of melatonin in promoting cucumber seed germination under high salinity by regulating energy production. Sci. Rep. 7:503.

Zhu, W., Smith, J.W., Huang, C. (2010). Mass spectrometry-based label-free quantitative proteomics. J. Biomed. Biotechnol. 840518. 